\title{
IL-17 Promotes Scar Formation by Inducing Macrophage Infiltration
}

Julei Zhang, ${ }^{*}$ Qiao Qiao, ${ }^{\dagger}$ Mengdong Liu, ${ }^{*}$ Ting He, ${ }^{*}$ Jihong Shi, ${ }^{*}$ Xiaozhi Bai, ${ }^{*}$ Yijie Zhang, ${ }^{*}$ Yan Li, ${ }^{*}$ Weixia Cai, * Shichao Han, ${ }^{*}$ Hao Guan, ${ }^{*}$ and Dahai Hu*

From the Department of Burns and Cutaneous Surgery, ${ }^{*}$ Xijing Hospital, and the Department of Implant Dentistry, ${ }^{\dagger}$ School of Stomatology, the Fourth Military Medical University, Xi'an, People's Republic of China

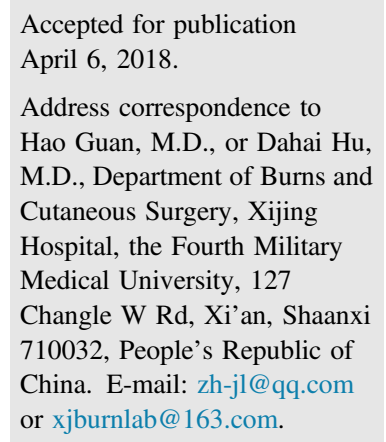

\begin{abstract}
Trauma or burn injuries that affect the deep dermis often produce a hypertrophic scar, which limits patients' joint movement and generates an aesthetic problem. Inflammation is believed to be one of the main pathogenic mechanisms. We found that IL-17 was increased in scar tissues from patients with hypertrophic scar compared with normal skin. Recombinant mouse IL-17 was subcutaneously injected into mice that underwent full-thickness excision surgery to investigate the role of IL-17 in scar formation. Mice stimulated with IL-17 showed aggravated fibrogenesis, delayed wound healing, and increased inflammation. In addition, macrophage infiltration was also increased. According to the results of the Transwell assay, IL-17 promoted macrophage infiltration through an indirect mechanism. After depleting macrophages with clodronate liposomes, the effect of IL-17 disappeared. Levels of monocyte chemotactic protein (MCP) 1, MCP2, and MCP3 (together referred to as MCPs) were increased by IL-17 stimulation. Bindarit (an inhibitor of MCPs) was used to verify the role of MCPs. In addition, the Ly6C-low macrophages were responsible for wound fibrogenesis in mice. In this study, we detected the increased levels of IL-17 for the first time and revealed that IL-17 induced the infiltration of a specific subtype of macrophages to aggravate fibrosis through an MCP-dependent mechanism. Thus, our results provide a better understanding of scar formation and new strategies for scar prevention. (Am J Pathol 2018, 188: 1693-1702; https://doi.org/10.1016/j.ajpath.2018.04.005)
\end{abstract}

Hypertrophic scars (HTSs) are the main chronic disease after burns, trauma, and surgery. Although HTSs are not life threatening, they annoy patients because of the limited joint movement and aesthetic problems, which pose a severe burden to the patients and society. ${ }^{1,2}$ Histopathologically, HTSs are characterized by the enhanced proliferation of fibroblasts, the increased transdifferentiation of fibroblasts into myofibroblasts, and the increased deposition of extracellular matrix proteins, such as type I collagen (COL-1). ${ }^{2,3}$ Excessive inflammation is believed to be one of the most important factors leading to the fibrogenesis of HTS. ${ }^{4,5}$ Current therapies for HTS include topical therapy, intralesional therapy, mechanical therapy, and surgical treatment. ${ }^{1,6}$ However, because of our insufficient understanding of the mechanism, the effects of these therapies are limited.

$\mathrm{IL}-17$ is a proinflammatory cytokine that is involved in inflammatory diseases, such as psoriasis and rheumatoid arthritis. ${ }^{7,8}$ Recently, IL-17 was reported to be involved in pulmonary fibrosis, ${ }^{9}$ bleomycin-induced systemic sclerosis, ${ }^{10}$ and liver fibrosis. ${ }^{11}$ However, the role of IL-17 in scar formation after cutaneous injury is still unclear.

Macrophages are an important type of innate immune cell that participate in antigen presentation, pathogen clearance, inflammatory reactions, and tissue regeneration. ${ }^{12-14}$ Depending on the microenvironment, macrophages might polarize to different subtypes, which play different roles in normal and pathologic processes. ${ }^{15,16}$ Increased infiltration of macrophages was recently observed in HTSs, ${ }^{17}$ but the reason and the exact effect of macrophages require further

\footnotetext{
Supported by the National Natural Science Foundation of China grants 81571914 (J.S.), 81601689 (T.H.), and 81772017 (D.H.), and The Burn Center of PLA, Department of Burns and Cutaneous Surgery, Xijing Hospital.

J.Z., Q.Q., and M.L. contributed equally to this work.

Disclosures: None declared.
} 
investigation. Ly6C is a surface cell marker that is used to identify different subsets of monocytes/macrophages. ${ }^{18}$ The Ly6C-high monocytes/macrophages are inflammatory cells that infiltrate into the inflammatory site, whereas the Ly6Clow monocytes/macrophages are a patrolling cell type that are responsible for replenishing resident macrophages. ${ }^{19}$ Ly6C-low macrophages have recently been reported to be responsible for remodeling liver fibrosis, ${ }^{19}$ whereas Ly6Clow macrophages have a profibrotic phenotype in a renal ischemia-reperfusion model. ${ }^{20}$ However, no study has focused on the infiltration and distribution of macrophages classified by Ly6C status in cutaneous fibrosis.

In this study, we investigated the role of IL-17 in scar formation. Increased levels of IL-17 were observed in HTS tissue compared with normal skin. In addition, in the mouse wound healing process, IL-17 promoted the formation of cutaneous scar with the help of increased levels of several chemokines and the infiltration of macrophages; different macrophage subtypes showed different functions. Our results disclosed the role of IL-17 in wound healing and the formation of cutaneous scar, providing new potential targets for preventing HTSs.

\section{Materials and Methods}

\section{Animals}

Adult male $\mathrm{BALB} / \mathrm{c}$ mice weighing 20 to $25 \mathrm{~g}$ were provided by the Experimental Animal Center of Fourth Military Medical University (Xi'an, China). The protocol was approved by the Animal Experiment Administration Committee of the Fourth Military Medical University. For establishing the cutaneous excision model, mice were anesthetized with an i.p. injection of sodium pentobarbital $(1 \%, 50 \mathrm{mg} / \mathrm{kg})$. Then, the dorsal skin was shaved, sterilized, and draped. A $1-\mathrm{cm} \times 1-\mathrm{cm}$ wound on the dorsal skin was generated using the standard template. The wound areas were imaged immediately and on days $1,2,3,4,6,8$, and 10 after surgery. Recombinant mouse IL-17 $(100 \mu \mathrm{L} ; 1 \mathrm{mg} / \mathrm{mL}$; R\&D Systems, Minneapolis, MN) was administered by s.c. injection immediately after the surgery and every 24 hours for the next 4 days. The mice were sacrificed at the designated time points ( 4 and 10 days after surgery), and the wound tissues were collected for further experiments.

\section{Culture of Scar and Skin Tissues and Fibroblasts}

HTS and normal skin tissues were collected from patients undergoing plastic surgery. All patients were informed about the purpose of the study and agreed to provide their excised tissues. For fibroblast cultures, neonatal mice were sacrificed and the dorsal skin was collected and digested with dispase $(0.25 \%)$ overnight to remove the epidermis. Then, the dermal tissues were cut into fragments with a diameter of $<1 \mathrm{~mm}$ and digested using type I collagenase $(0.2 \% \mathrm{w} / \mathrm{v}$; SigmaAldrich, St. Louis, MO) for 2 hours at $37^{\circ} \mathrm{C}$. The divided cells were cultured in Dulbecco's modified Eagle's medium (Gibco, San Diego, CA) supplemented with $10 \%$ fetal bovine serum (Gibco), $100 \mathrm{U} / \mathrm{mL}$ penicillin, and $100 \mathrm{U} / \mathrm{mL}$ streptomycin (BasalMedia, Shanghai, China) at $37^{\circ} \mathrm{C}$ in a $5 \%$ (v/v) $\mathrm{CO}_{2}$-humidified incubator. Cells at passages 3 to 5 were used in the experiments.

\section{Macrophage Depletion}

Clodronate liposomes (CELs; Liposoma, Amsterdam, the Netherlands) were used to deplete macrophages, according to the manufacturer's instructions. Briefly, mice were injected with CELs $(100 \mu \mathrm{L})$ through the tail vein 24 hours before and 24 and 72 hours after surgery. The efficacy of macrophage depletion was confirmed by immunohistochemistry (IHC) and flow cytometry.

\section{Cell Migration Assay}

A total of $1 \times 10^{6}$ RAW 264.7 macrophages in $200 \mu \mathrm{L}$ of serum-free Dulbecco's modified Eagle's medium was seeded into the upper chamber of Transwells $(8-\mu \mathrm{m}$ pore size; Millipore, Darmstadt, Germany), and the lower chamber contained $500 \mu \mathrm{L}$ of Dulbecco's modified Eagle's medium supplemented with $10 \%$ fetal bovine serum. For the cell coculture assay, mouse skin fibroblasts were seeded into the lower chamber and were allowed to grow to confluence before the upper chamber was inserted. For stimulation, 100 $\mathrm{ng} / \mathrm{mL}$ recombinant mouse IL-17 was added to the lower chamber. Twenty-four hours after the insertion of the upper chamber, the cells remaining in the upper chamber were wiped off with a cotton swab and the migrated cells were fixed with $4 \%$ paraformaldehyde for 15 minutes and stained with crystal violet for 30 minutes. Then, the migrated cells were observed and imaged using FSX100 Bio Imaging Navigator (Olympus, Tokyo, Japan).

\section{Inhibitor Administration}

Bindarit and Y-320 (both from Selleck, Houston, TX) were used to inhibit monocyte chemotactic protein (MCP) 1,2 , or 3 or IL-17, respectively. Bindarit was administered to each animal at a dose of $50 \mathrm{mg} / \mathrm{kg}$ per day by oral gavage. Y-320 was administered to each animal at a dose of $3 \mathrm{mg} / \mathrm{kg}$ per day by oral gavage. Both inhibitors were dissolved in dimethyl sulfoxide, according to the manufacturer's instructions. Both inhibitors were administered for 5 consecutive days after surgery.

\section{Western Blot Analysis}

For the detection of COL-1, $50 \mu \mathrm{g}$ of total proteins was separated using SDS-PAGE and transferred onto polyvinylidene fluoride membranes. Membranes were blocked with 5\% nonfat milk for 2 hours and incubated with primary antibodies against IL-17 (1:1000; Proteintech, Wuhan, 
China), COL-1 (1:1000; Abcam, Cambridge, UK), and glyceraldehyde-3-phosphate dehydrogenase (1:1000; CST, Boston, MA) overnight at $4^{\circ} \mathrm{C}$. Then, the membranes were incubated with the secondary antibodies (1:3000; Boster, Wuhan, China) for 1 hour at room temperature. Protein bands were visualized with an ECL Kit (Millipore) and imaged using a FluorChem FC system (Alpha Innotech, San Leandro, CA).

\section{Quantitative Real-Time RT-PCR}

RNA (500 ng) was extracted using an RNA-Isolation Kit (Takara, Dalian, China) and reverse transcribed using a Prime Script RT Reagent Kit (Takara). The cDNAs were then amplified using PrimeScript RT Master Mix (Takara) in a CFX Connect Real-Time PCR Detection System (BioRad Laboratories, Hercules, CA). The primers sequences are listed below. The results were normalized to the level of glyceraldehyde-3-phosphate dehydrogenase. Primer sequences were as follows: COL-1, 5'-TGACTGGAAGAGCGGAGAGT (forward) and $5^{\prime}$-ATCCATCGGTCATGCTCTCT-3' (reverse); glyceraldehyde-3-phosphate dehydrogenase, 5'GTGTTCCTACCCCCAATGTG-3' (forward) and 5'-CATCGAAGGTGGAAGAGTGG-3' (reverse); interferon- $\gamma$, $5^{\prime}$-TTGAAAGACAATCAGGCCATC-3' (forward) and $5^{\prime}$ TGAGCTCATTGAATGCTTGG- $3^{\prime}$ (reverse); IL-1 $\beta, 5^{\prime}$ GGGCCTCAAAGGAAAGAATC- $3^{\prime}$ (forward) and $5^{\prime}$-TACCAGTTGGGGAACTCTGC-3' (reverse); tumor necrosis factor- $\alpha, 5^{\prime}$-CGTCAGCCGATTTGCTATCT- $3^{\prime}$ (forward) and $5^{\prime}$ CTTGGGCAGATTGACCTCAG-3' (reverse); IL-6, 5'-GGGACTGATGCTGGTGACAA-3' (forward) and 5'-TCCACGATTTCCCAGAGAACA-3' (reverse); MCP1, 5'-GTTAACGCCCCACTCACCTG-3' (forward) and 5'-CCCATTCCTTCTTGGGGTCA-3' (reverse); MCP2, 5'-TTCTTTGCCTGCTGCTCATA-3' (forward) and $5^{\prime}$-ATACCCTGCTTGGTCTGGAA$3^{\prime}$ (reverse); MCP3, 5'-AATGCATCCACATGCTGCTA-3' (forward) and $5^{\prime}$-GGAGTTGGGGTTTTCATGTC-3' (reverse); and IL-17, 5'-ACTACCTCAACCGTTCCACG-3' (forward) and 5'-ATGTGGTGGTCCAGCTTTCC-3' (reverse).

\section{Masson's Trichrome Staining and IHC}

Tissues from patients and the wound area of mice were fixed with $4 \%$ paraformaldehyde for 24 hours, decalcified, embedded in paraffin blocks, cut into tissue sections $(4 \mu \mathrm{m}$ thick), and stained with Masson's Stain Kit (Nanjing Jiancheng, Nanjing, China), according to the manufacturer's instructions. For IHC staining, SPlink Detection Kits (ZSGB-BIO, Beijing, China) were used, according to the manufacturer's instructions. The primary antibodies used for IHC included IL-17 (1:100; Proteintech), COL-1 (1:100; Abcam), and F4/80 (1:50; Abcam).

\section{Flow Cytometry}

Tissues were digested using collagenase type I $(0.2 \% \mathrm{w} / \mathrm{v}$; Sigma-Aldrich) to obtain dissociated single cells. Cells were blocked with an anti-CD16/CD32 antibody (BD Pharmingen, San Jose, CA). Next, the cells were incubated with antimouse CD11b- phosphatidylethanolamine, Ly6C-PerCpCy5.5 (both from eBioscience, San Diego, CA), and F4/ 80-APC (Biolegend, San Diego, CA) antibodies at $4^{\circ} \mathrm{C}$ for 30 minutes. Then, the cells were washed and analyzed using a BD ACCURI C6 flow cytometer (BD Pharmingen). $\mathrm{CD} 11 \mathrm{~b}^{+} \mathrm{F} 4 / 80^{+}$cells were considered macrophages.

\section{Enzyme-Linked Immunosorbent Assay}

Anti-mouse IL-17, MCP1, MCP2, MCP3, IL-6, interferon$\gamma$, and tumor necrosis factor- $\alpha$ enzyme-linked immunosorbent assay kits (Wayen Biotechnologies, Shanghai, China) were used to assay scar cytokine levels, according to the manufacturer's instructions.

\section{Statistical Analysis}

Statistical analyses of data from two groups were performed using $t$-test or one-way analysis of variance for data for

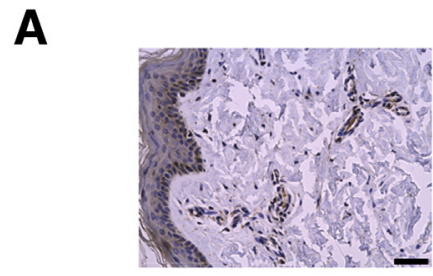

NS

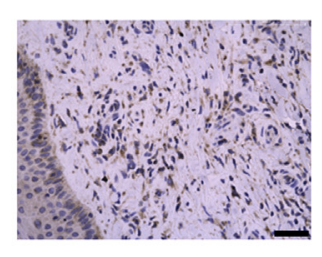

HTS
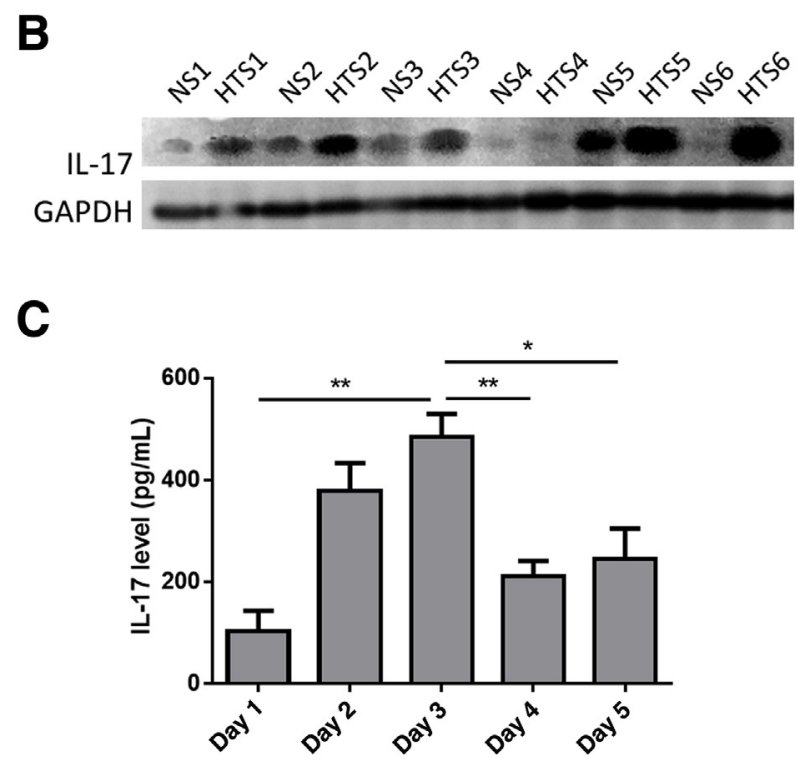

Figure 1 IL-17 levels in normal skin, in hypertrophic scar (HTS) tissues, and in the mouse cutaneous wound healing process. A and B: Representative images showing IL-17 levels in HTS and normal skin (NS) tissues detected using immunohistochemistry (A) and Western blotting (B). C: The full-thickness excision model was built, and protein levels of the IL-17 in wound tissues were analyzed by enzyme-linked immunosorbent assay, over the next 5 days. $n=6(\mathbf{A}$ and $\mathbf{B}) ; n=5(\mathbf{C}) .{ }^{*} P<0.05,{ }^{*} P>0.01$. Scale bars $=50 \mu \mathrm{m}(\mathbf{A}) . \mathrm{GAPDH}$, glyceraldehyde-3-phosphate dehydrogenase. 
multiple groups. All analyses were performed using GraphPad Prism software version 5.00 (GraphPad Software Inc., La Jolla, CA). All data are presented as the means \pm SEM. $P<0.05$ was considered statistically significant.

\section{Results}

\section{IL-17 Levels Are Increased in HTS Tissues and in the Cutaneous Wound Healing Process in Mice}

Patients with burn injuries, one of the main causes of HTSs, exhibit high levels of IL-17. ${ }^{21}$ HTS and normal skin tissues were collected from patients receiving plastic surgery to investigate IL-17 levels. Higher IL-17 levels were detected in HTS tissues than in normal skin (Figure 1, A and B). Then, the full-thick dorsal cutaneous wound model was built to investigate IL-17 expression in mice. During the wound healing process, the mRNA and protein levels of IL-17 were increased in the initial stage and peaked on day 3 (Figure 1C).

\section{IL-17 Promotes Scar Formation in Mice}

To test whether persistently elevated IL-17 levels aggravate the fibrogenesis process in HTSs, mice were subcutaneously injected with recombinant mouse IL-17 during the wound healing process. The healing speed was significantly delayed by IL-17 compared with the control group (Figure 2, A and B). The extracellular matrix deposition within the wound area was detected by Masson's trichrome staining and showed an increase on the $10^{\text {th }}$ day after the IL17 injection (Figure 2C). In addition, levels of the COL-1 mRNA and protein were significantly increased (Figure 2D). Thus, the increased level of IL-17 might be one factor that promotes HTS formation. Inflammation is considered to play a key role in scar formation; therefore, the inflammatory reaction was examined after the IL-17 injection. During the wound healing process, levels of the tumor necrosis factor- $\alpha$, interferon- $\gamma$, and IL-6 proteins were all increased (Figure 2E) after IL-17 stimulation. Thus, IL-17 may promote scar formation through its proinflammatory and profibrotic effects.

\section{IL-17 Promotes Macrophage Infiltration}

IL-17 was shown to promote inflammatory reactions by inducing the infiltration of inflammatory cells, particularly neutrophils, and cytokine production. ${ }^{7}$ As shown in previous studies, HTSs contain a greater number of infiltrating macrophages ${ }^{22}$; therefore, it was determined whether IL-17 promotes HTS formation by recruiting macrophages. Thus, macrophage infiltration was evaluated using flow cytometry and IHC in wound areas, and it was found that IL-17
A

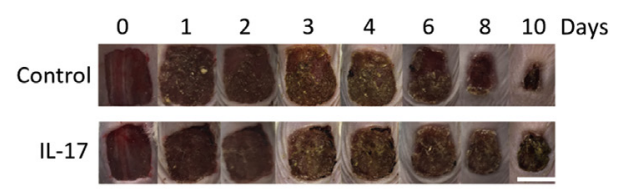

C
Control

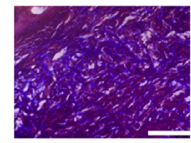

IL-17

$\mathbf{E}$

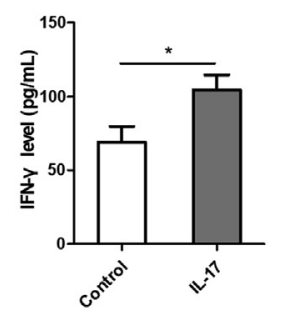

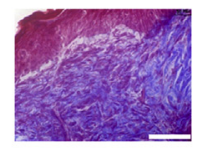

B

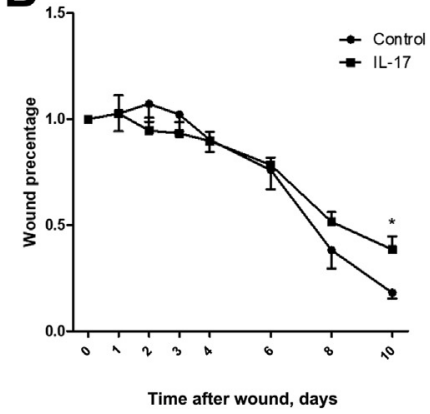

D

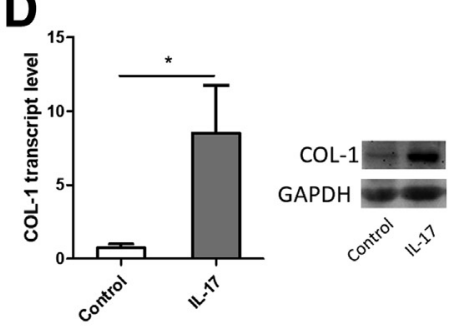

Figure 2 The effect of IL-17 on the wound healing process in mice. The full-thickness excision model was built, the wound healing speed was recorded (A and $\mathbf{B})$, collagen deposition on day 10 was examined using Masson's trichrome staining (C), levels of the type I collagen (COL-1) mRNA and protein on day 10 were detected using quantitative real-time RT-PCR and Western blotting, respectively (D), and the protein levels of several inflammatory factors were detected on day 4 using enzyme-linked immunosorbent assays (E). $n=5$ $(\mathbf{A}-\mathbf{E}) .{ }^{*} P<0.05$ versus control (t-test). Scale bars: $1 \mathrm{~cm} \mathrm{(A);50} \mu \mathrm{m}($ C). GAPDH, glyceraldehyde3-phosphate dehydrogenase; IFN- $\gamma$, interferon- $\gamma$; TNF- $\alpha$, tumor necrosis factor- $\alpha$.
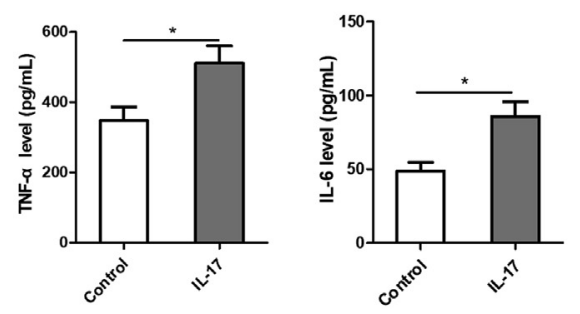
increased the number of macrophages, as expected (Figure 3, A and B). A co-culture system based on Transwells was used to investigate whether IL-17 directly recruited macrophages. IL-17 alone was not able to recruit RAW274.7 macrophages to migrate from the upper chamber to the lower chamber (Figure 3C). Given the complicated microenvironment in the wound area, we hypothesized that IL-17 might induce macrophage infiltration through an indirect mechanism. Thus, fibroblasts, the most abundant cell type in wound areas, were seeded into the lower chamber of the Transwell system and grown in culture medium containing IL-17, whereas RAW264.7 macrophages were seeded into the upper chamber. The combination of fibroblasts and IL-17 obviously induced the migration of RAW264.7 macrophages from the upper chamber to the lower chamber (Figure 3, C and D). Therefore, IL-17 indirectly promotes the infiltration of RAW264.7 cells.

\section{Macrophage Depletion Abrogates the Profibrotic Effect of IL-17}

Because macrophages participate in the fibrotic process, it was tested whether IL-17-induced macrophages promote scar formation. The increased number of infiltrating macrophages colocalized with COL-1 in the wound bed, implying a relationship between macrophages and fibrosis (Figure 4A). Then, CEL was used to specifically deplete monocytes/macrophages. The effect of the depletion was confirmed by IHC and flow cytometry, because
IL-17-induced macrophage infiltration was almost eliminated by CELs (Figure 3, A and B). Using this strategy, it was confirmed that IL-17-induced fibrosis required macrophages, because the depletion of macrophages significantly abrogated the effect of IL-17 on extracellular matrix deposition (Figure 4B) and COL-1 expression (Figure 4C). However, the wound healing speed was still delayed by IL-17 and was even much slower in response to CEL administration than in the control group (Figure 4, D and E). Therefore, fibrosis, but not the wound healing speed, correlates with macrophage infiltration.

\section{IL-17 Recruits Macrophages by Inducing MCPs}

IL-17 might promote macrophage infiltration by inducing the production of other soluble factors. Thus, the levels of several chemokines that induce macrophage infiltration were examined, and it was found that the wound site showed increased levels of MCP1, MCP2, and MCP3 (Figure 5A) on the fourth day after IL-17 stimulation. Then, Bindarit, an inhibitor of MCPs, was used to identify the role of MCPs in the wound healing and scar formation processes. The effect was evaluated using enzyme-linked immunosorbent assays (Figure 5A). After the inhibition of MCPs, macrophage infiltration was reduced (Figure 5, B and $\mathrm{C}$ ), followed by decreased collagen expression and deposition (Figure 5, D and E). Therefore, the profibrotic effect of IL-17 partially depended on MCPs. In addition, the wound healing speed was increased after Bindarit administration (Figure 5, F and G). Interestingly, the

A

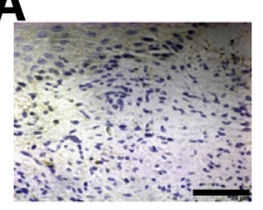

Control

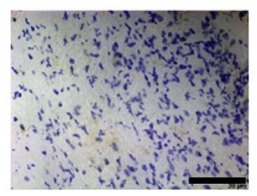

CEL

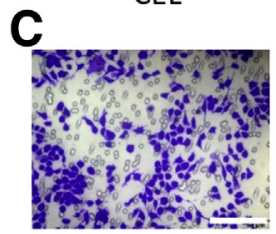

Control

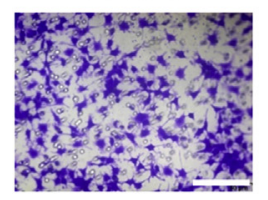

$\mathrm{NFb}$

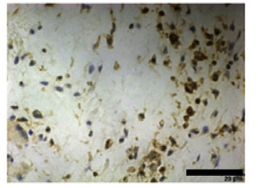

IL-17

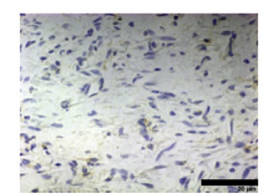

CEL+IL-17

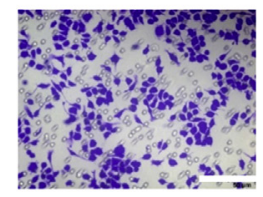

IL-17

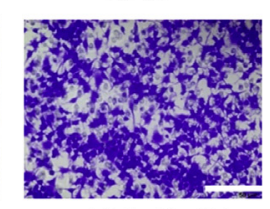

$\mathrm{NFb+IL-17}$
B
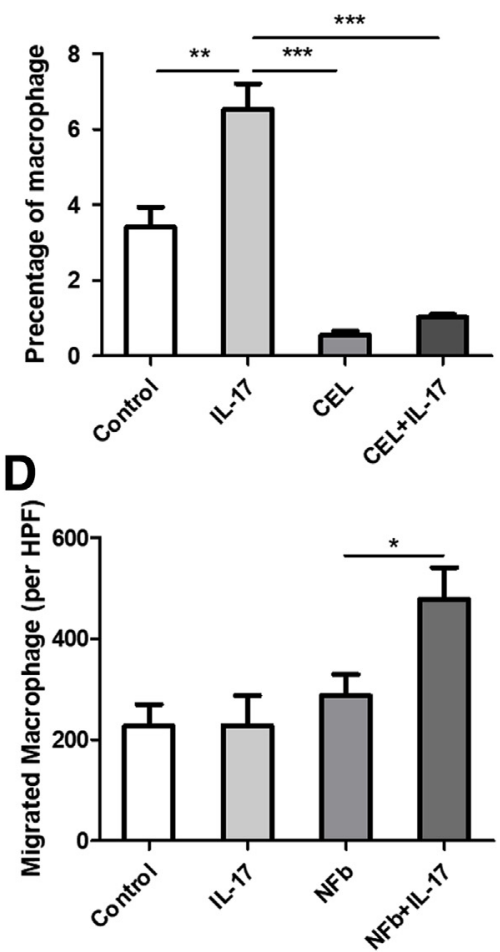

Figure 3 The effect of IL-17 on inducing macrophage infiltration. $\mathbf{A}$ and $\mathbf{B}$ : Mice were treated with IL-17 and with or without clodronate liposome (CEL) after surgery, and the infiltration of macrophages into the wound area was evaluated on day 10 after surgery using immunohistochemistry (A) and flow cytometry (B). C and D: The effect of IL-17 on inducing macrophage infiltration was evaluated using a Transwell co-culture system. Macrophages were stimulated with IL-17 and cultured with or without normal fibroblasts (NFbs) for 24 hours. $n=5$ (A-D). ${ }^{*} P<0.05,{ }^{* *} P<0.01$, and ${ }^{* * * P}<0.001$. Scale bars $=50 \mu \mathrm{m}(\mathbf{A}$ and $\mathbf{C})$. HPF, high-power field. 


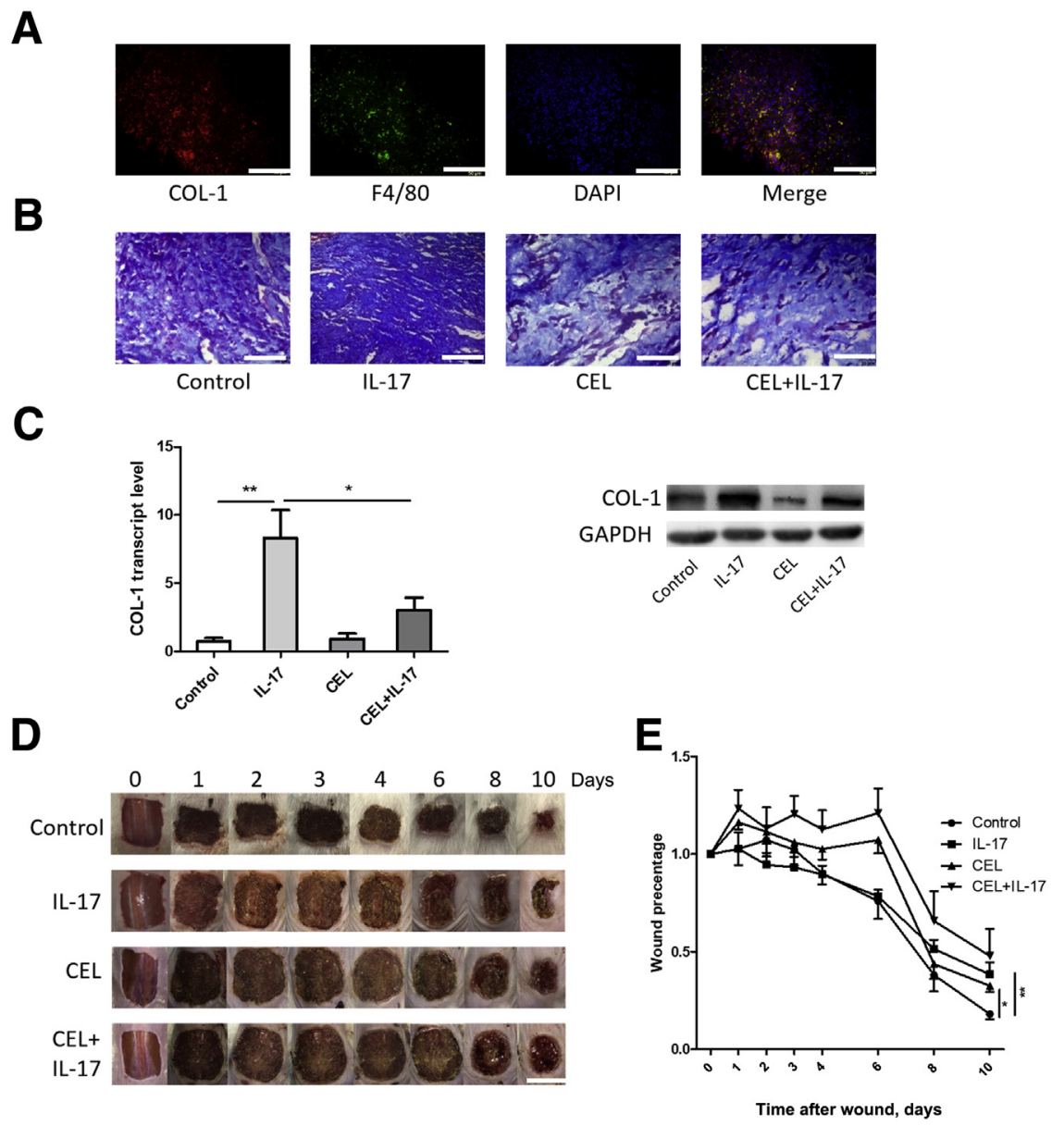

Figure 4 The effect of macrophage depletion on IL-17-induced skin fibrosis and delayed wound healing. A: Type I collagen (COL-1) and macrophage localization in wound tissues was detected on day 10 using immunofluorescence staining. DAPI was used for nuclear staining. B-E: Mice were treated with IL-17 and with or without clodronate liposomes (CELs) after surgery, collagen deposition on day 10 was examined using Masson's trichrome staining (B), levels of the COL-1 mRNA and protein were detected on day 10 using quantitative real-time RT-PCR and Western blotting, respectively $(\mathbf{C})$, and the wound healing speed was recorded (D and $\mathbf{E}) . n=5(\mathbf{A}-\mathbf{E}) .{ }^{*} P<0.05$, ${ }^{* *} P<0.01$. Scale bars: $50 \mu \mathrm{m}(\mathbf{A}$ and $\mathbf{B}) ; 1 \mathrm{~cm}$ (D). GAPDH, glyceraldehyde-3-phosphate dehydrogenase.

administration of Y-320, an inhibitor of IL-17, did not significantly alter fibrosis (data not shown) or the wound healing speed at the last checkpoint (day 10) (Figure 5, F and G). However, the wound healing speed was significantly increased in the first few days after the administration of Y-320. On the basis of these data and the results shown in Figure 1C, Y-320 might inhibit the increased IL-17 levels produced in the initial stage of wound healing. Thus, these results again proved that the increased IL-17 levels delayed the wound healing speed.

\section{Ly6C-Low Macrophages Are Responsible for Fibrosis}

Ly6C, a surface marker of monocytes/macrophages, was used to investigate the subtype of macrophages induced by IL-17 and obtain a better understanding of the roles of IL-17 and macrophages in cutaneous wound healing and fibrogenesis. After the administration of IL-17, the wound area showed a significantly increased number of the Ly6C-low subtype (Figure 6). However, the administration of CEL and Y-320 decreased the number of Ly6C-low macrophages. Thus, the changes in the numbers of Ly6C-low subtype macrophage were consistent with the changes in the degree of fibrosis (Figure 4B, Figure 5D, and Figure 6). However, the delayed wound healing speed was not consistent with the fibrosis and the numbers of the Ly6C-low subtype, because the aforementioned results (Figure 4) showed that CEL resulted in delayed wound healing and alleviated fibrosis. In conclusion, the fibrosis occurring during the wound healing process is at least partially mediated by Ly6C-low macrophages, but the reason for the change in healing speed requires further investigation.

\section{Discussion}

Excess inflammation is believed to be one of the main causes of HTS. Anti-inflammatory agents have proved to be a promising therapeutic strategy for preventing scar formation. ${ }^{4,5,23-27}$ However, the underlying mechanism is still unclear. Macrophages, an immune cell type required for tissue repair and regeneration, have drawn increasing attention to their roles in fibrotic diseases. ${ }^{28}$ Recent studies have observed increased numbers of macrophages in HTS tissues, ${ }^{29}$ and depletion of macrophages using gene editing technology alleviates scar formation in a mouse HTS model. ${ }^{29}$ However, the HTS model used in this experiment was generated in athymic nude mice, which lack the natural 
A

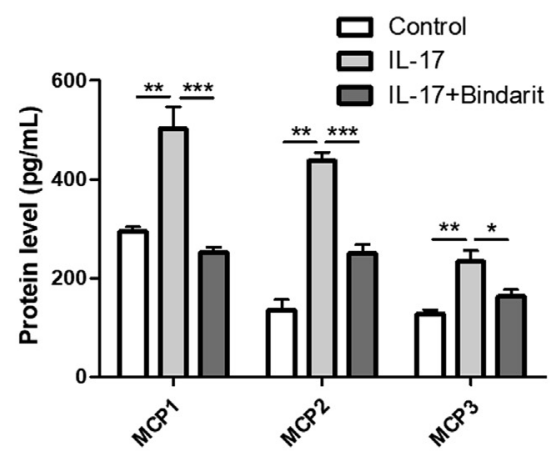

D

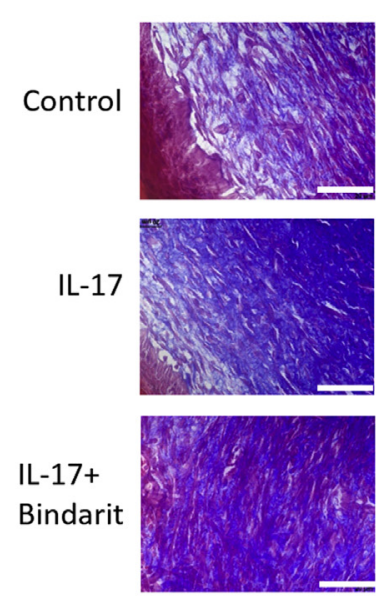

$\mathbf{F}$

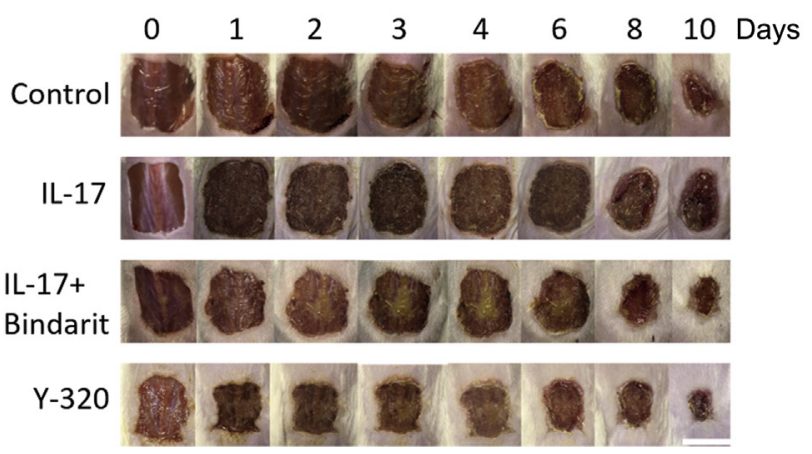

B

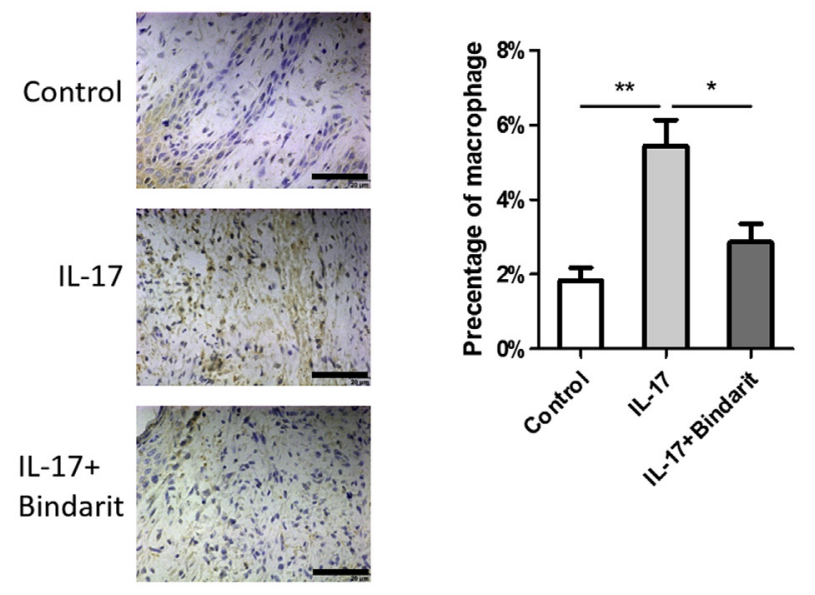

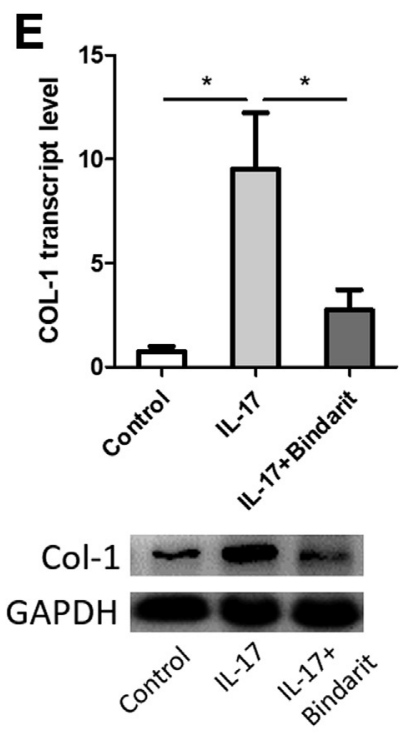

G

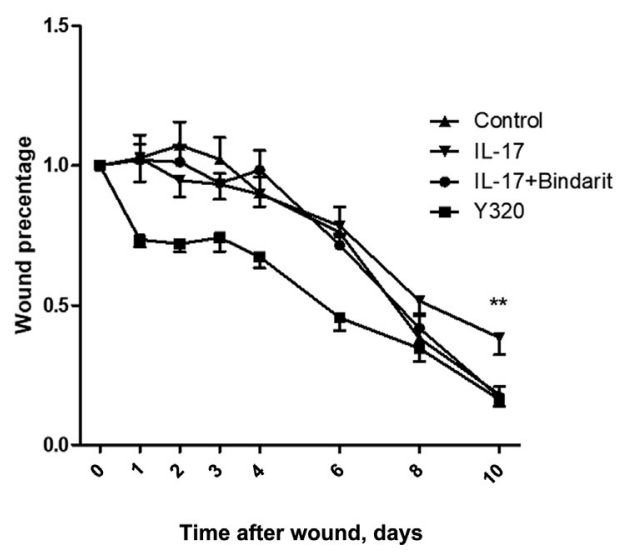

Figure 5 The effect of a monocyte chemotactic protein (MCP) inhibitor on IL-17-induced skin fibrosis and delayed wound healing. Mice were treated by IL-17 and with or without Bindarit (MCP inhibitor) after surgery, levels of the MCP proteins (MCP1, MCP2, and MCP3) were detected on day 4 using enzyme-linked immunosorbent assays (A), the infiltration of macrophages into the wound area on day 10 after surgery was evaluated using immunohistochemistry (B) and flow cytometry (C), collagen deposition on day 10 was examined using Masson's trichrome staining (D), levels of the type I collagen (COL-1) mRNA and protein were detected on day 10 using quantitative real-time RT-PCR and Western blotting, respectively $(\mathbf{E})$, and the wound healing speed was recorded $(\mathbf{F}$ and $\mathbf{G}) . n=5(\mathbf{A}-\mathbf{G}) .{ }^{*} P<0.05,{ }^{*} P<0.01$, and ${ }^{* * *} P<0.001$ versus control (analysis of variance). Scale bars: $50 \mu \mathrm{m}(\mathbf{B}$ and $\mathbf{D}) ; 1 \mathrm{~cm}(\mathbf{F})$. GAPDH, glyceraldehyde-3-phosphate dehydrogenase. 

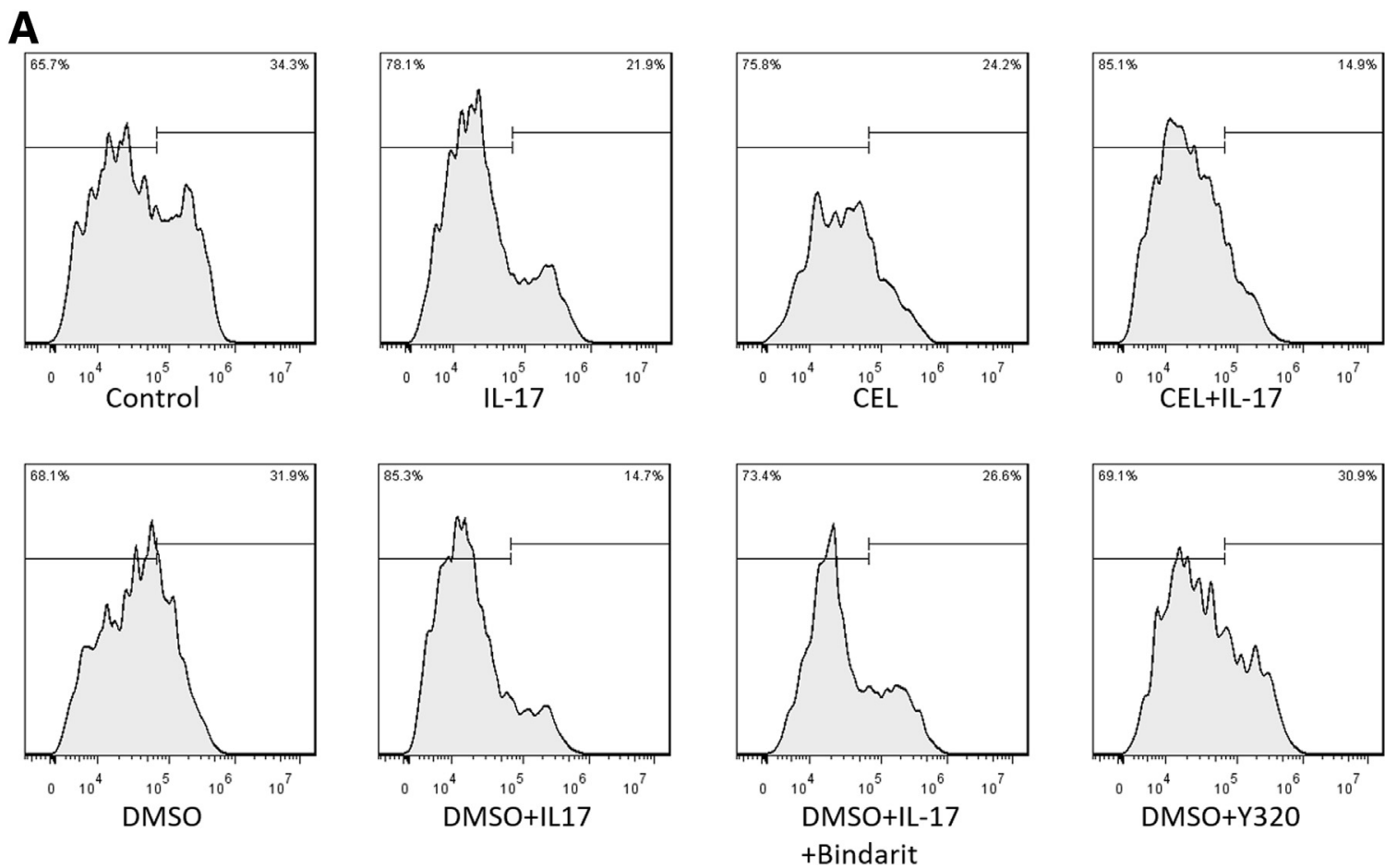

B

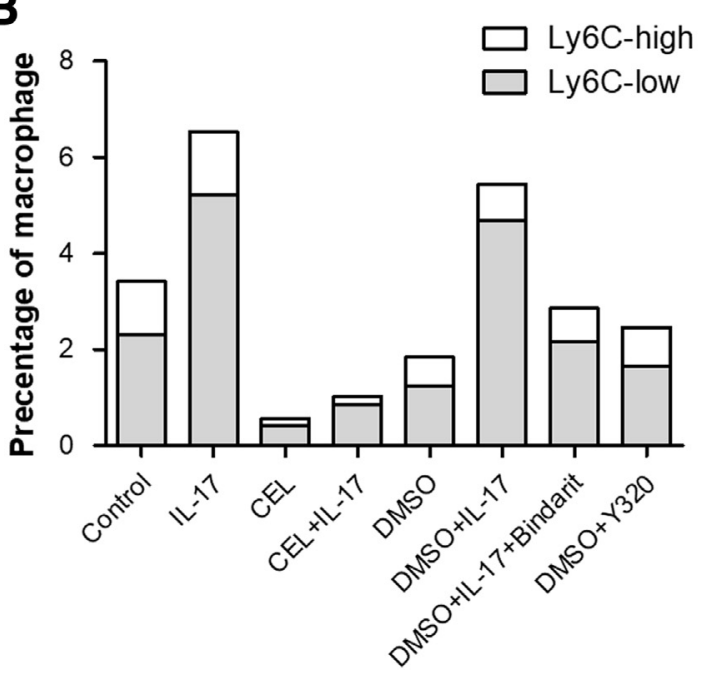

C

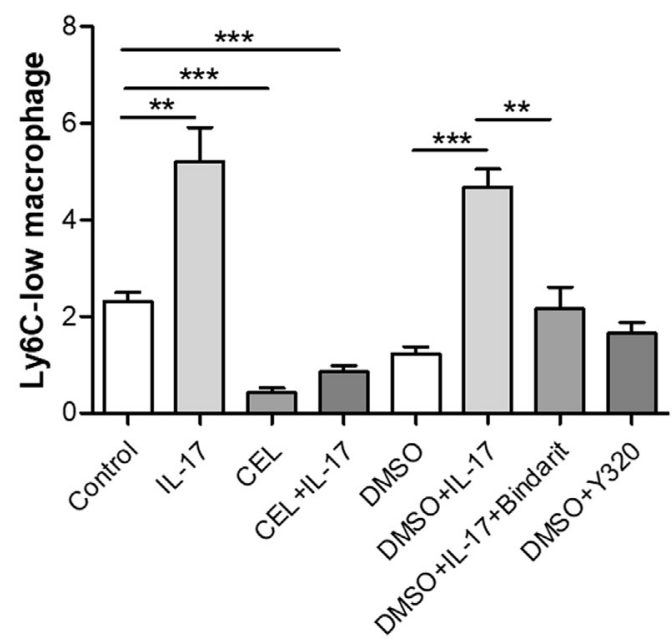

Figure 6 Changes in the number of the Ly6C-low subtype of macrophages. Mice were stimulated with IL-17 along with or without Bindarit, clodronate liposomes (CELS), or Y-320 (IL-17 inhibitor), and the total number and number of the Ly6C-low subtype of macrophages were analyzed using flow cytometry. $n=5(\mathbf{A}-\mathbf{C}) .{ }^{* *} P<0.01,{ }^{* *} P<0.001$. DMS0, dimethyl sulfoxide.

inflammatory and immune reactions present in human patients. Therefore, additional research is needed to obtain a better understanding of the role of macrophages in the development of HTSs.

IL-17 participates in several fibrotic diseases, including lung, liver, and skin fibrosis, by participating in the local inflammatory response, but the role of macrophages in these processes is unclear. ${ }^{9-11}$ In this study, we reported an increased level of IL-17 in HTSs for the first time and found that IL-17 may induce macrophage infiltration to promote scar formation in mice, providing new insights into HTSs and other fibrotic diseases.
In clinical practice, a delayed wound healing speed is considered one of the most important signs that predict HTS formation. Deep wounds, which need a longer recovery period, are highly related to HTSs. ${ }^{30}$ Consistent with this phenomenon, IL-17 significantly decreased the healing speed of the wound and subsequently exerted a profibrotic effect. However, after macrophage depletion, the healing speed was delayed again. This result was unexpected, because we postulated that the IL-17 increase in macrophage infiltration was the main reason for the delayed wound healing. In addition, Bindarit exerted a different effect on wound healing than CELs. This finding might be 
explained by the different mechanisms by which CEL and Bindarit reduce the recruitment of macrophages. CEL targets macrophages undergoing phagocytosis, whereas Bindarit targets macrophages induced by MCP1, MCP2, and MCP3. Thus, these two subtypes of macrophage might have different functions, which requires further investigation. All these questions prompted us to investigate potential changes in the subtype of macrophages that could explain the observed results. Interestingly, the surface marker Ly6C has drawn scientists' attention because it is believed to play a key role in distinguishing macrophage subsets with different functions, but the effect seems to depend on the organ and disease. ${ }^{18-20}$ In this study, the number of Ly6Clow macrophages was consistent with the fibrosis of the wound area, showing the same trend in a renal ischemiareperfusion model. Therefore, we speculated that Ly6Clow macrophages are responsible for the fibrosis in the skin wound healing process. However, the wound healing speed did not match the number of Ly6C-low macrophages or the fibrosis level, as shown in the CEL group. This result differs from a recent study showing that Ly6C-low macrophages participate in wound closure, and this subtype is missing in subjects with diabetes, resulting in delayed wound healing. This discrepancy might be explained by the special microenvironment in subjects with diabetes. Thus, the functions of different subtypes of macrophages depend on the disease, and additional research is required to completely elucidate the wound healing process. In addition, this classification of macrophages provides new insights into the recently discussed relationship between inflammation, macrophages, and HTSs, because recently two articles declare that patients with HTSs exhibit decreased inflammation and increased numbers of macrophages, ${ }^{17,31,32}$ findings that differ from the aforementioned research. Thus, scientists should further investigate the subtypes of macrophages and their exact roles in the formation of HTSs and other fibrotic diseases.

In conclusion, our study found increased levels of IL-17 in HTS tissues and proved that IL-17 could promote cutaneous wound fibrogenesis in a mouse model by increasing the number of Ly6C-low macrophages through an MCPdependent mechanism.

\section{Supplemental Data}

Supplemental material for this article can be found at https://doi.org/10.1016/j.ajpath.2018.04.005.

\section{References}

1. Block L, Gosain A, King TW: Emerging therapies for scar prevention. Adv Wound Care (New Rochelle) 2015, 10:607-614

2. Chiang RS, Borovikova AA, King K, Banyard DA, Lalezari S, Toranto JD, Paydar KZ, Wirth GA, Evans GR, Widgerow AD: Current concepts related to hypertrophic scarring in burn injuries. Wound Repair Regen 2016, 3:466-477
3. Zhang J, Li Y, Bai X, Li Y, Shi J, Hu D: Recent advances in hypertrophic scar. Histol Histopathol 2018, 33:27-39

4. Qian LW, Fourcaudot AB, Yamane K, You T, Chan RK, Leung KP: Exacerbated and prolonged inflammation impairs wound healing and increases scarring. Wound Repair Regen 2016, 1:26-34

5. Wong VW, Rustad KC, Akaishi S, Sorkin M, Glotzbach JP, Januszyk M, Nelson ER, Levi K, Paterno J, Vial IN, Kuang AA, Longaker MT, Gurtner GC: Focal adhesion kinase links mechanical force to skin fibrosis via inflammatory signaling. Nat Med 2012, 1:148-152

6. Gauglitz GG, Korting HC, Pavicic T, Ruzicka T, Jeschke MG: Hypertrophic scarring and keloids: pathomechanisms and current and emerging treatment strategies. Mol Med 2011, 1-2:113-125

7. Miossec P, Kolls JK: Targeting IL-17 and TH17 cells in chronic inflammation. Nat Rev Drug Discov 2012, 10:763-776

8. Korn T, Bettelli E, Oukka M, Kuchroo VK: IL-17 and Th17 cells. Annu Rev Immunol 2009, 27:485-517

9. Wilson MS, Madala SK, Ramalingam TR, Gochuico BR, Rosas IO, Cheever AW, Wynn TA: Bleomycin and IL-1beta-mediated pulmonary fibrosis is IL-17A dependent. J Exp Med 2010, 3:535-552

10. Okamoto Y, Hasegawa M, Matsushita T, Hamaguchi Y, Huu DL, Iwakura Y, Fujimoto M, Takehara K: Potential roles of interleukin$17 \mathrm{~A}$ in the development of skin fibrosis in mice. Arthritis Rheum 2012, 11:3726-3735

11. Meng F, Wang K, Aoyama T, Grivennikov SI, Paik Y, Scholten D, Cong M, Iwaisako K, Liu X, Zhang M, Osterreicher CH, Stickel F, Ley K, Brenner DA, Kisseleva T: Interleukin-17 signaling in inflammatory, Kupffer cells, and hepatic stellate cells exacerbates liver fibrosis in mice. Gastroenterology 2012, 3:765-776

12. Lech M, Anders HJ: Macrophages and fibrosis: how resident and infiltrating mononuclear phagocytes orchestrate all phases of tissue injury and repair. Biochim Biophys Acta 2013, 7:989-997

13. Das A, Sinha M, Datta S, Abas M, Chaffee S, Sen CK, Roy S: Monocyte and macrophage plasticity in tissue repair and regeneration. Am J Pathol 2015, 10:2596-2606

14. Lucas T, Waisman A, Ranjan R, Roes J, Krieg T, Muller W, Roers A, Eming SA: Differential roles of macrophages in diverse phases of skin repair. J Immunol 2010, 7:3964-3977

15. Sica A, Erreni M, Allavena P, Porta C: Macrophage polarization in pathology. Cell Mol Life Sci 2015, 21:4111-4126

16. Motwani MP, Gilroy DW: Macrophage development and polarization in chronic inflammation. Semin Immunol 2015, 4:257-266

17. van den Broek LJ, van der Veer WM, de Jong EH, Gibbs S, Niessen FB: Suppressed inflammatory gene expression during human hypertrophic scar compared to normotrophic scar formation. Exp Dermatol 2015, 8:623-629

18. Epelman S, Lavine KJ, Beaudin AE, Sojka DK, Carrero JA, Calderon B, Brija T, Gautier EL, Ivanov S, Satpathy AT, Schilling JD, Schwendener R, Sergin I, Razani B, Forsberg EC, Yokoyama WM, Unanue ER, Colonna M, Randolph GJ, Mann DL: Embryonic and adult-derived resident cardiac macrophages are maintained through distinct mechanisms at steady state and during inflammation. Immunity 2014, 1:91-104

19. Ramachandran P, Pellicoro A, Vernon MA, Boulter L, Aucott RL, Ali A, Hartland SN, Snowdon VK, Cappon A, Gordon-Walker TT, Williams MJ, Dunbar DR, Manning JR, van Rooijen N, Fallowfield JA, Forbes SJ, Iredale JP: Differential Ly-6C expression identifies the recruited macrophage phenotype, which orchestrates the regression of murine liver fibrosis. Proc Natl Acad Sci U S A 2012, 46: E3186-E3195

20. Clements M, Gershenovich M, Chaber C, Campos-Rivera J, Du P, Zhang M, Ledbetter S, Zuk A: Differential Ly6C expression after renal ischemia-reperfusion identifies unique macrophage populations. J Am Soc Nephrol 2016, 1:159-170

21. Sasaki JR, Zhang Q, Schwacha MG: Burn induces a Th-17 inflammatory response at the injury site. Burns 2011, 4:646-651

22. Amini-Nik S, Cambridge E, Yu W, Guo A, Whetstone H, Nadesan P, Poon R, Hinz B, Alman BA: Beta-Catenin-regulated myeloid cell 
adhesion and migration determine wound healing. J Clin Invest 2014, 6:2599-2610

23. Shi J, Li J, Guan H, Cai W, Bai X, Fang X, Hu X, Wang Y, Wang H, Zheng Z, Su L, Hu D, Zhu X: Anti-fibrotic actions of interleukin-10 against hypertrophic scarring by activation of PI3K/AKT and STAT3 signaling pathways in scar-forming fibroblasts. Plos One 2014, 5:e98228

24. Cremers NA, Suttorp M, Gerritsen MM, Wong RJ, van Run-van BC, van Dam GM, Brouwer KM, Kuijpers-Jagtman AM, Carels CE, Lundvig DM, Wagener FA: Mechanical stress changes the complex interplay between HO-1, inflammation and fibrosis, during excisional wound repair. Front Med (Lausanne) 2015, 2:86

25. Ud-Din S, Volk SW, Bayat A: Regenerative healing, scar-free healing and scar formation across the species: current concepts and future perspectives. Exp Dermatol 2014, 9:615-619

26. Wang H, Chen Z, Li XJ, Ma L, Tang YL: Anti-inflammatory cytokine TSG-6 inhibits hypertrophic scar formation in a rabbit ear model. Eur J Pharmacol 2015, 751:42-49

27. Shi J, Wang H, Guan H, Shi S, Li Y, Wu X, Li N, Yang C, Bai X, Cai W, Yang F, Wang X, Su L, Zheng Z, Hu D: IL10 inhibits starvation-induced autophagy in hypertrophic scar fibroblasts via cross talk between the IL10-IL10R-STAT3 and IL10-AKT-mTOR pathways. Cell Death Dis 2016, 3:e2133

28. Borthwick LA, Barron L, Hart KM, Vannella KM, Thompson RW, Oland S, Cheever A, Sciurba J, Ramalingam TR, Fisher AJ, Wynn TA: Macrophages are critical to the maintenance of IL-13-dependent lung inflammation and fibrosis. Mucosal Immunol 2015, 1:38-55

29. Zhu Z, Ding J, Ma Z, Iwashina T, Tredget EE: Systemic depletion of macrophages in the subacute phase of wound healing reduces hypertrophic scar formation. Wound Repair Regen 2016, 4: 644-656

30. Penn JW, Grobbelaar AO, Rolfe KJ: The role of the TGF-beta family in wound healing, burns and scarring: a review. Int J Burns Trauma 2012, 1:18-28

31. Kwon SH, Gurtner GC: Is early inflammation good or bad? linking early immune changes to hypertrophic scarring. Exp Dermatol 2016 , 26:133-134

32. Butzelaar L, Schooneman DP, Soykan EA, Talhout W, Ulrich MM, van den Broek LJ, Gibbs S, Beelen RH, Mink VDMA, Niessen FB: Inhibited early immunologic response is associated with hypertrophic scarring. Exp Dermatol 2016, 10:797-804 